\title{
Screening the Efficacy of Compounds from Ghee to Control Cancer: An In silico Approach
}

\author{
Abinaya Madhavan 1, Priya Sankar ${ }^{1}$, Karunya Jenin Ravindranath ${ }^{1}$, Ranjani Soundhararajan 1(D), \\ Hemalatha Srinivasan 1 ,*(i) \\ 1 School of Life Sciences, B. S. Abdur Rahman Crescent Institute of Science and Technology, Vandalur, India \\ * Correspondence: hemalatha.sls@bsauniv.ac.in;
}

Received: 15.01.2021; Revised: 25.02 .2021 ; Accepted: 2

\begin{abstract}
Ghee, common fat, is being consumed by many people worldwide as a dietary. Research shows that the consumption of ghee provides health benefits, including anti-cancer effect. Numerous fatty acids in ghee are found to be effective in killing cancer cells and in aiding various physiological processes. Compounds present in ghee are analyzed for their anti-cancer properties through in silico analysis. The selected fatty acids in the ghee are analyzed through molecular docking and molecular dynamics, and the interactions of these compounds with the target proteins are determined to interpret the affinity of their interaction. The interaction between the fatty acids presents in the home-made ghee, and the target proteins (AIF, PTEN, BAX, BCL2, and CASPASE9) highlights the importance and efficiency of the home-made ghee in providing anti-cancer effects. This study also provides evidence that consuming home-made ghee both as a part of the diet and on an empty stomach might increase the preventive possibilities for the incidence of cancer, and also ghee consumption might reduce a person's susceptibility for inheriting some of the cancer types like breast and colon cancer which needs confirmation through further in vitro research.
\end{abstract}

Keywords: ghee; anti-cancer effects; fatty acids;in silico analysis; molecular docking; molecular dynamics.

(c) 2021 by the authors. This article is an open-access article distributed under the terms and conditions of the Creative Commons Attribution (CC BY) license (https://creativecommons.org/licenses/by/4.0/).

\section{Introduction}

Ghee is a class of clarified butter with nutritional value, providing the energy of about $469 \mathrm{KJ}$ per tablespoon and a flavonoid with a good aroma. Ghee has small, medium, long-chain, and some unsaturated fatty acids. It also contains high levels of CLA- conjugated linoleic acid that has been shown to inhibit the growth of tumor cells of breast cancer [1,2]. Moreover, CLA is also found to play an important role in antioxidant homeostasis and possesses immunostimulatory properties [3]. Ghee is also highly rich in butyric acid, a short-chain fatty acid $[4,5]$. In the intestine, the fibers are converted into butyric acid, and it provides around 60$70 \%$ of the total energy necessary for the intestine's colon cells. A healthy body thus makes its form of ghee. Further studies also show that an adequate amount of butyric acid production supports the production of killer T cells in the gut that enhances the functioning of the immune system [6].

Cancer is an abnormal growth of cells caused by multiple changes in gene expression, leading to an imbalance in cellular proliferation and apoptosis (programmed cell death), resulting in the growth of the population of cells that invades the nearby tissues and metastasize to distant sites. These cancer cells have specific hallmarks that make them strong in survival 
and immortality, such as self-sufficiency of growth signals, insensitivity to anti-growth signals, continuous angiogenesis, evading programmed cell death, invasion and metastasis, unlimited replicative potential, genome instability, and various types of mutations, etc. [7]. Home-made ghee is found to have numerous fatty acids, and hence this experiment aims to determine the possibility of each of the selected components present in ghee for its anti-tumor effects without causing any adverse side-effects. Surgery, radiotherapy, chemotherapy are some of the commonly used strategies for treating various types of cancers, and among these, radiotherapy and chemotherapy are increasingly used for treating different metastatic cancers. However, the drugs used for radiotherapy and chemotherapy shows the incidence of various side effects, which include fatigue, anemia, hair loss, nausea, vomiting, diarrhea, skin problems, nerve issues, muscle deformities, kidney and bladder irritation, and various fertility and sexual problems [8]. Moreover, commercially available anti-cancer drugs can induce toxicity in the human body and might lead to some serious consequences such as alopecia, depression in the bone marrow, loss of immunity and self-confidence, and also a considerable decline in the WBC count [9]. Cancer cells are also smart enough to use various escape mechanisms for survival and enhanced proliferation by inhibiting apoptosis and also by developing multiple drug resistance capabilities, ultimately resulting in the failure of these conventional treatments. Thus, an efficient drug would be a "molecule" that is highly specific in killing only tumor cells leaving healthy cells unharmed and one with very minimal side-effects. Research has also proven over time that many natural products serve this purpose [10-12]. Thus, this current study aims to screen the efficiency of fatty acids present in home-made ghee that might serve as a novel compound with anti-cancer properties.

\section{Materials and Methods}

The in silico assay was performed using the Tripos' SYBYL-X Version 1.3 [13-15] that has the ability to perform multiple as well as individual scoring functions and ranking. Docking was performed between the ligands of interest with each of the target proteins. Pubchem and Protein Data Bank are used for retrieving the structures, while Pymol viewer is used for result analysis.

\subsection{Preparation of target proteins.}

The 3D structures of the target proteins AIF- Apoptosis inducible factor, BAX- Bcl2 Associated X protein, Bcl2- B cell lymphoma2, Caspase 9, PTEN- phosphatase, and tensile homolog with the structure IDs $1 \mathrm{M} 6 \mathrm{I}, 1 \mathrm{~F} 16,1 \mathrm{G} 5 \mathrm{M}, 1 \mathrm{NW} 9$ respectively were retrieved from the protein data bank in the PDB format.

\subsection{Preparation of ligands of interest.}

The three dimensional structures of the ligands of interest Arachidic Acid (structure 2D_CID_10467), butyric acid (structure3D_CID_8892), Capric acid (structure3D_CID_2969), Caproic acid (structure3D_CID_8892), Caprylic acid (structure3D_CID_379), Cis linoleic acid (structure3D_CID_6431005), Cis palmitoleic acid (structure3D_CID_445638), Conjugated linoleic acid (structure3D_CID_5280644), Hexadecanoic acid (structure3D_CID_985), Myristic acid (structure3D_CID11005), Pentadecanoic acid (structure3D_CID13899), Stearic acid (structure3D_CID_5281), Trans oleic acid (structure3D_CID_637517) structures were retrieved from Pubchem. 


\subsection{Molecular docking.}

\subsubsection{Target protein processing.}

The three-dimensional structure of the target proteins- AIF (FAD1449), BAX (OXT192), Bcl2 (OXT207), Caspase9 (OXT416), p53, PTEN (ZN501) are acquired from the protein data bank and then given as input in the Swiss PDB viewer to remove the unwanted, already existing residues and ligands. The processed structures of the protein are then saved as a separate file.

\subsubsection{Screening of rule of five.}

The factor determining if a particular molecule is a drug or non-drug based on the complex balance of different molecular properties is referred to as drug likeliness. Some of these molecular properties include lipophilicity, electronic distribution, hydrogen bonding, molecular size, and flexibility. The presence of various pharmacophoric features also influences a molecule's behavior in a living organism, which includes characteristics such as transport, affinity to proteins, reactivity, toxicity, metabolic stability, etc. Although absorption and bioavailability are two separate parameters, information about absorption could be used for bioavailability modeling. A way for screening the compounds with probable absorption problems is known as Lipinski's rule of five or Pfizer's rule of five or rule of five. This rule states that the poor absorption or permeation of a drug is more probable when the chemical structure fulfills two or more of the following: Molecular weight is greater than 500 Daltons, Hydrogen bond donors greater than 5, Hydrogen bond acceptors greater than 10, Calculated $\log \mathrm{P}$ value greater than 5, Molecular refractivity not between 40-130.

\subsubsection{Docking.}

The molecular docking is performed in the Sybyl Docking suite with the default parameters. The target proteins are first downloaded in the pdb file format, which needs to be converted to SFXC file format for performing molecular docking in sybyl [13-15]. Once the target protein is defined in the sybyl, it is then subjected to the preparation step, where it needs to be converted into Mol2 file format.

Once the target protein is converted into Mol2 file format, the protomol is generated, and the active site is noted, and hence the protein is prepared in SFXC file format. The ligands of interest, which are in sdf file format, are selected and converted to Mol2 file format. The protein in the SFXC format and the ligand in Mol2 format is chosen in the docking panel, and a docking run is performed. Once the docking is completed, the results are automatically saved in the default directory chosen initially.

\subsubsection{Radius of gyration.}

The Radius of Gyration helps to determine the compactness of the protein structure. If a protein is folded in a stable manner, a relatively steady value of Radius of Gyration can be observed. However, if the protein is found to be unstable, the radius of gyration is found to vary accordingly. This helps to determine the stability of the interaction of ligands of interest with the target proteins. 
In molecular docking and dynamic studies, a stable interaction is considered to be one, where The Root Mean Square Deviation does not exceed $2 \AA$ from the normal structure, and the Radius of Gyration is maintained at $20 \AA \AA$.

\section{Results and Discussion}

The results file of the docked protein and ligand are then opened in the Pymol Viewer, and the amino acid residues to which the ligand has bonded, the bond length, the poses of the binding are recorded and saved. Top one pose is selected based on the CScore (that should be $>4$ as the maximum value of CScore is 5), polar value (that denotes the polar contact), and the Total Score (that denotes the scoring of all the parameters) (Tables 1-6).

The scores (high polar values and total score) and high affinity (less bond length) show that when people continuously consume ghee, the compounds in the ghee (fatty acids) can enhance apoptosis and decrease the surviving ability of the cells when there is an incidence of cancer. Apoptosis inducible factor (AIF) can induce apoptosis by triggering DNA fragmentation and chromatin condensation [15]. Apoptosis is initiated when mitochondria release AIF, which exists through the mitochondrial space into the cytosol, reaches the cell nucleus, and provides signals to the cell to condense its chromosomes fragments its DNA. Ghee has a high smoke point of about $250^{\circ} \mathrm{c}$, and so when it is used in cooking, it does not breakdown into free radicals, unlike some cooking oils.

The high score and affinity of the ghee compounds with tumor suppressor protein PTEN indicate that the phosphatase protein product involved in the cell cycle regulation will prevent or inhibit the cells from growing and dividing too rapidly when there is the onset of cancer and its prevalence. The higher level of interaction of the ghee's fatty acids can be considered sufficient to kill tumor cells as it inhibits one of the important signaling pathways that support the growth of the cancer cells- AKT signaling pathway.

The Capric, Caproic, and Caprylic acids constitute about $15 \%$ of goat milk and are shown to have higher medicinal properties in the goat milk [16-18]. These fatty acids are found to down-regulate the $\mathrm{p}$ genes such as CDK2 (Cyclin-dependent kinase 2), CDK4(Cyclin dependent kinase 4), CKS1B(CDC 28 protein kinase 1B), CCNA2(Cyclin A2), and CCND1(Cyclin D) in the HCT116 cells that are essential for the cell cycle division and cellular progression in colon cancer cells [16-18]. It is also observed that these three fatty acids upregulate the GADD45a gene that plays an important role in the programmed cell death in colon cancer cells. It is also found that the Capric acid, Caproic acid, Caprylic acids down-regulate the expression of CKS1B, CCNA2, CCND1, and up-regulate NR4A1 gene (Peroxiredoxin1) and P21 (Cyclin-dependent kinase inhibitor1) that are required for the apoptosis in skin cancer cells [19].

From figures 1-5, it was observed that stearic acid, conjugated linoleic acid (CLA), butyric acid had proper interactions with both AIF and PTEN protein suggesting that there might be a decrease in breast cancer risks on the consumption of CLA. However, some research studies show no significant changes in the breast cancer risks even after the consumption of CLA [20]. Stearic acid is found to have a total score of about 10.8346 and 9.5522, the polar value of 4.0271 and 4.0514, global Cscore of 4 and 5 with Apoptosis inducible factor and Phosphatase tensile homolog, respectively, which supports the fact that stearic acid present in the home-made ghee can influence through cancer negative regulation. 

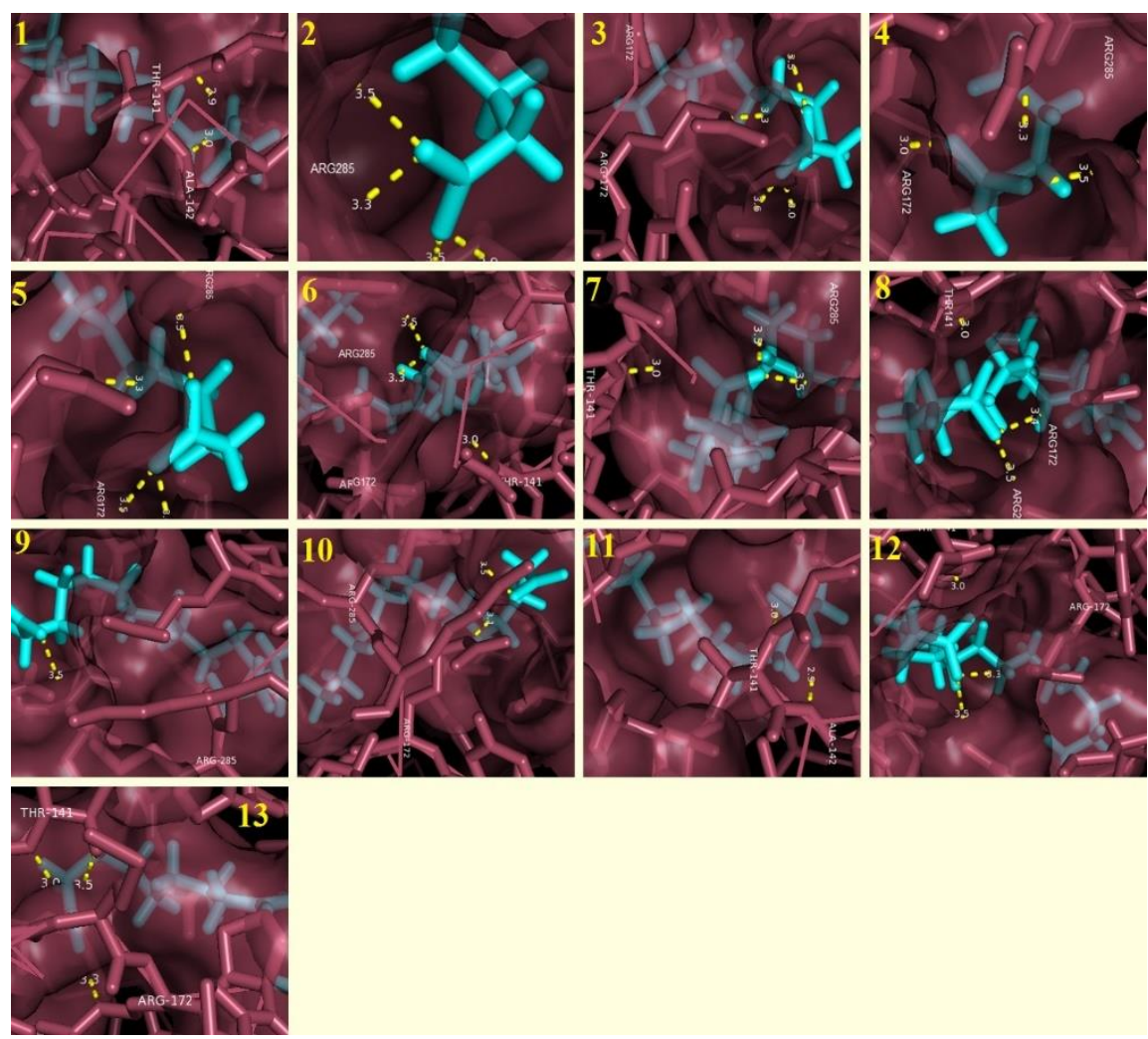

Figure 1. Interaction of fatty acids (ligands) from ghee with target protein- AIF (Apoptosis inducible factor). The ligands used are 1 - Arachidic Acid; 2 - Butyric Acid; 3 - Capric Acid; 4 - Caproic Acid; 5 - Caprylic Acid; 6 Cis linoleic Acid; 7 - Cis palmitoleic Acid; 8 - Conjugated linoleic Acid; 9 - Hexadecanoic Acid; 10 - Myristic Acid; 11 - Pentadecanoic Acid; 12 - Stearic Acid; 13 - Trans Oleic Acid.
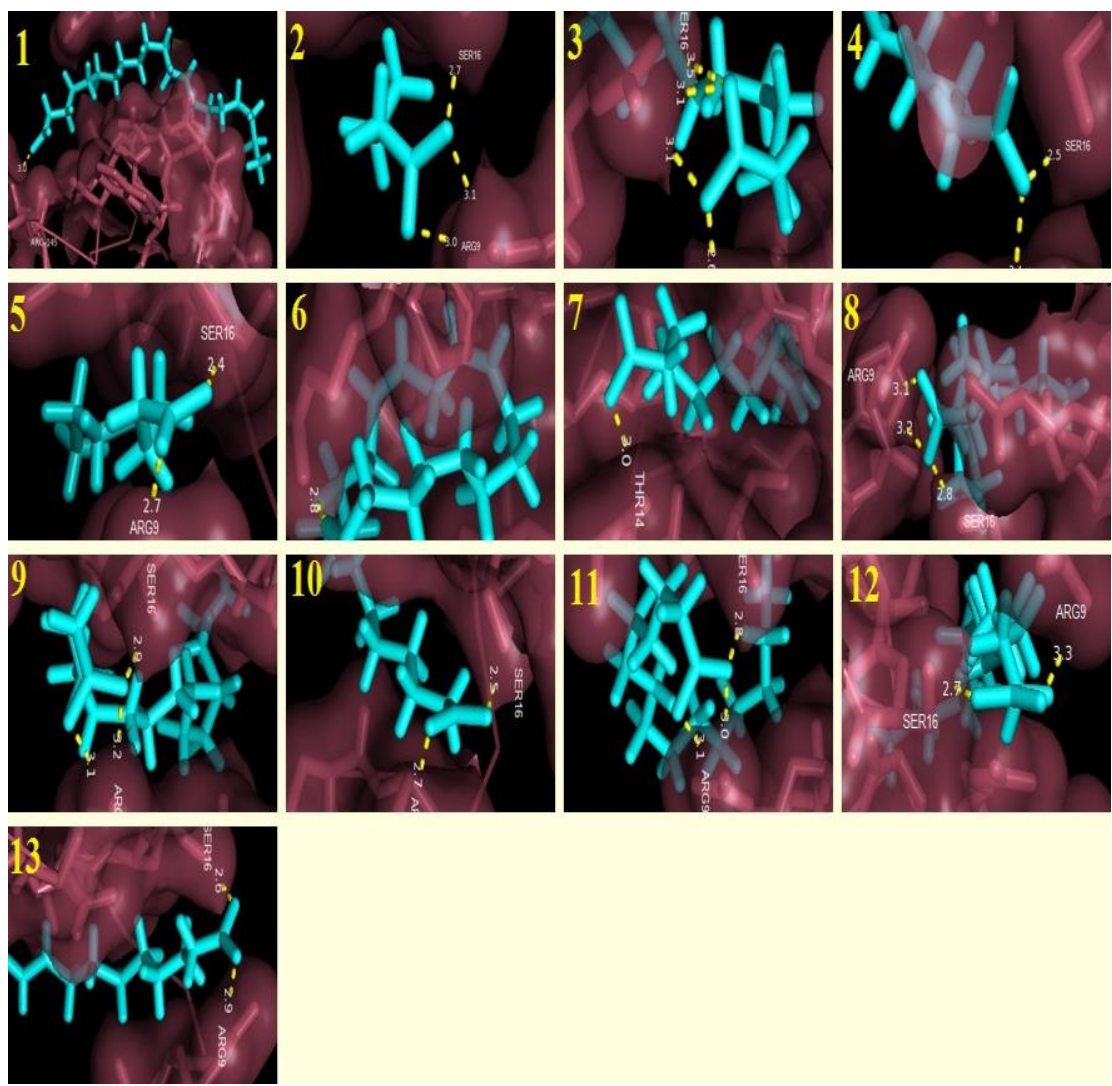

Figure 2. Interaction of fatty acids (ligands) with target protein-PTEN (Phosphatase and tensile homolog). The ligands used are 1 - Arachidic Acid; 2 - Butyric Acid; 3 - Capric Acid; 4 - Caproic Acid; 5 - Caprylic Acid; 6 Cis linoleic Acid; 7 - Cis palmitoleic Acid; 8 - Conjugated linoleic Acid; 9 - Hexadecanoic Acid; 10 - Myristic Acid; 11 - Pentadecanoic Acid; 12 - Stearic Acid; 13 - Trans Oleic Acid. 

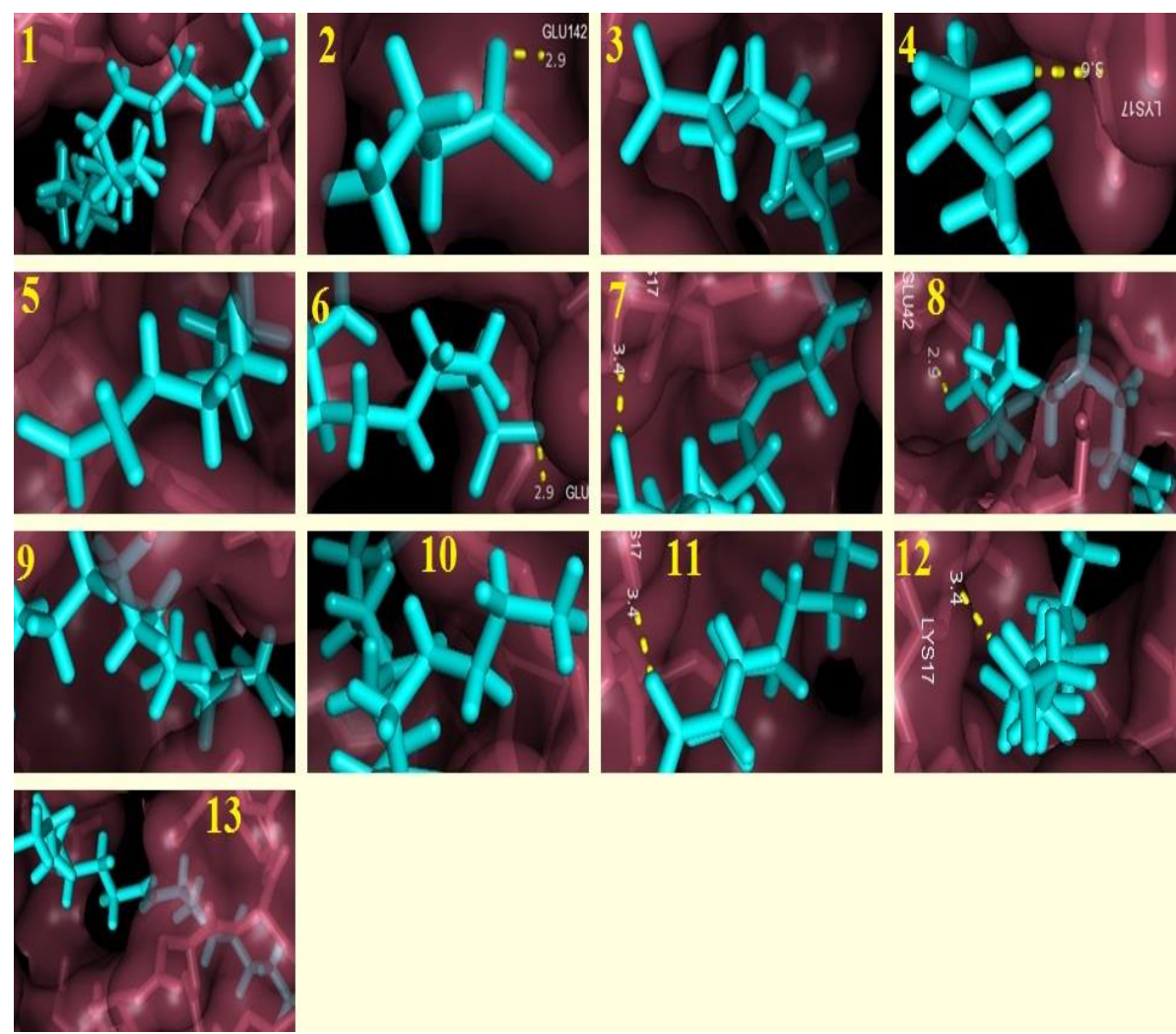

Figure 3. Interaction of fatty acids (ligands) with target protein-BAX (Bcl2 Associated Protein X) The ligands used are 1 - Arachidic Acid; 2 - Butyric Acid; 3 - Capric Acid; 4 - Caproic Acid; 5 - Caprylic Acid; 6 - Cis linoleic Acid; 7 - Cis palmitoleic Acid; 8 - Conjugated linoleic Acid; 9 - Hexadecanoic Acid; 10 - Myristic Acid; 11 Pentadecanoic Acid; 12 - Stearic Acid; 13 - Trans Oleic Acid.
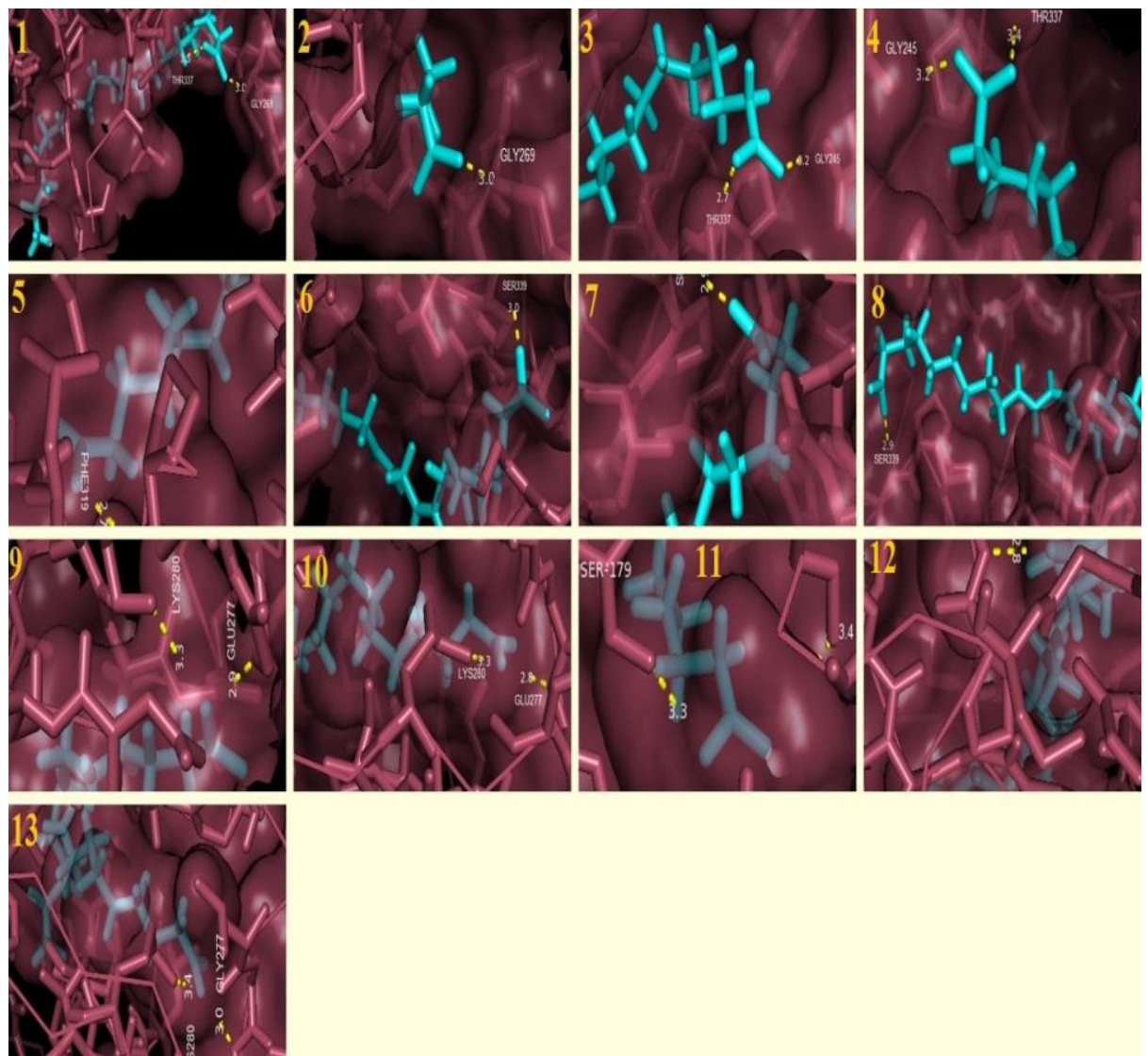

Figure 4. Interaction of fatty acids (ligands) with target protein-Bcl2 (B-cell Lymphoma2). The ligands used are 1 - Arachidic Acid; 2 - Butyric Acid; 3 - Capric Acid; 4 - Caproic Acid; 5 - Caprylic Acid; 6 - Cis linoleic Acid; 7 - Cis palmitoleic Acid; 8 - Conjugated linoleic Acid; 9 - Hexadecanoic Acid; 10 - Myristic Acid; 11 Pentadecanoic Acid; 12 - Stearic Acid; 13 - Trans Oleic Acid. 

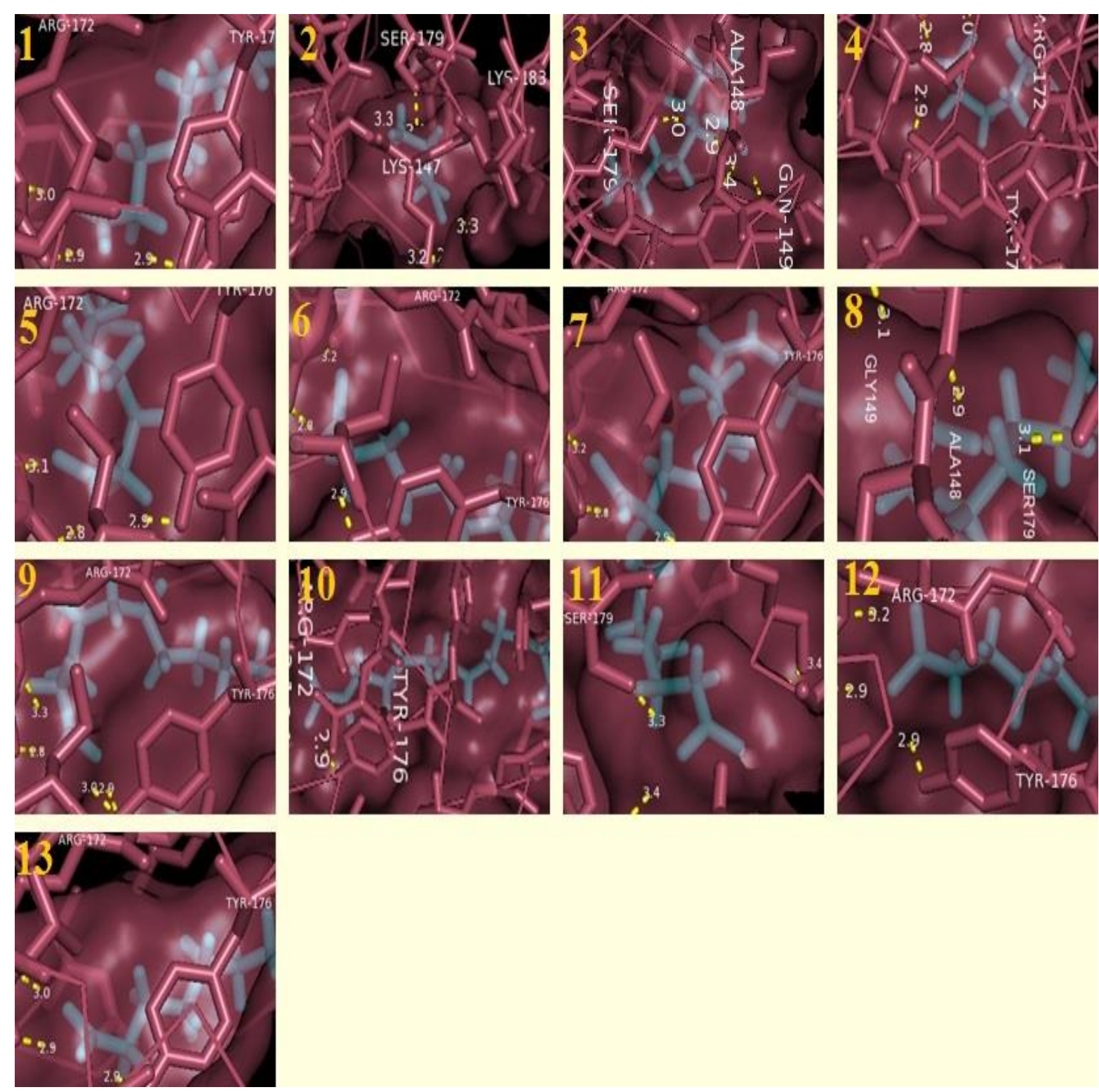

Figure 5. Interaction of fatty acids (ligands) with target protein- CASPASE9. The ligands used are 1 - Arachidic Acid; 2 - Butyric Acid; 3 - Capric Acid; 4 - Caproic Acid; 5 - Caprylic Acid; 6 - Cis linoleic Acid; 7 - Cis palmitoleic Acid; 8 - Conjugated linoleic Acid; 9 - Hexadecanoic Acid; 10 - Myristic Acid; 11 - Pentadecanoic Acid; 12 - Stearic Acid; 13 - Trans Oleic Acid.

Table 1. Scoring of the interaction between ligands and target protein AIF.

\begin{tabular}{l|c|c|c} 
Ligands with AIF & Total Score & Polar & Global C Score \\
\hline Arachidic acid & 11.6067 & 1.8928 & 4 \\
\hline Butyric acid & 4.8926 & 4.1092 & 4 \\
\hline Capric acid & 7.6191 & 4.0084 & 2 \\
\hline Caproic acid & 5.5630 & 4.0350 & 2 \\
\hline Caprylic acid & 6.7209 & 4.0491 & 4 \\
\hline Cis Linoleic acid & 9.7453 & 4.0685 & 4 \\
\hline Cis Palmitoleic acid & 9.7421 & 4.0860 & 4 \\
\hline Conjugated Linolenic acid & 10.0548 & 3.8376 & 5 \\
\hline Hexadecanoic acid & 9.6860 & 2.6327 & 3 \\
\hline Myristic acid & 7.8267 & 2.6886 & 5 \\
\hline Pentadecanoic acid & 8.6898 & 2.8894 & 4 \\
\hline Stearic acid & 10.8346 & 4.0271 & 5
\end{tabular}


Table 2. Scoring of the interaction between ligands and target protein PTEN.

\begin{tabular}{|c|c|c|c|}
\hline Ligands with PTEN & Total Score & Polar & Global C Score \\
\hline Arachidic acid & 8.6787 & 3.8809 & 3 \\
\hline Butyric acid & 5.5546 & 3.9412 & 4 \\
\hline Capric acid & 6.2026 & 3.9483 & 5 \\
\hline Caproic acid & 5.6917 & 4.3202 & 4 \\
\hline Caprylic acid & 6.1991 & 4.4019 & 2 \\
\hline Cis Linoleic acid & 8.7182 & 4.3560 & 2 \\
\hline Cis Palmitoleic acid & 8.4899 & 4.3277 & 5 \\
\hline Conjugated Linolenic acid & 7.9091 & 3.8668 & 5 \\
\hline Hexadecanoic acid & 8.3762 & 3.7421 & 3 \\
\hline Myristic acid & 8.1685 & 3.9790 & 3 \\
\hline Pentadecanoic acid & 8.2185 & 3.7263 & 3 \\
\hline Stearic acid & 9.5522 & 4.0514 & 5 \\
\hline Trans oleic acid & 8.4785 & 3.9981 & 5 \\
\hline
\end{tabular}

Table 3. Scoring of the interaction between ligands and target protein BAX.

\begin{tabular}{|c|c|c|c|}
\hline Ligands with Bax & Total Score & Polar & Global C Score \\
\hline Arachidic acid & 4.8274 & 1.4219 & 3 \\
\hline Butyric acid & 2.3011 & 2.5324 & 5 \\
\hline Capric acid & 3.5583 & 1.9800 & 2 \\
\hline Caproic acid & 2.4170 & 0.9806 & 3 \\
\hline Caprylic acid & 3.3324 & 1.1381 & 4 \\
\hline Cis Linoleic acid & 5.8649 & 1.4785 & 5 \\
\hline Cis Palmitoleic acid & 5.5493 & 1.4132 & 3 \\
\hline Conjugated Linolenic acid & 5.3940 & 2.6535 & 5 \\
\hline Hexadecanoic acid & 4.9192 & 2.1309 & 4 \\
\hline Myristic acid & 4.5071 & 1.2545 & 4 \\
\hline Pentadecanoic acid & 4.1030 & 2.6237 & 3 \\
\hline Stearic acid & 4.2156 & 1.6220 & 4 \\
\hline Trans oleic acid & 4.3572 & 1.4753 & 4 \\
\hline
\end{tabular}

Table 4. Scoring of the interaction between ligands and target protein $\mathrm{Bcl} 2$.

\begin{tabular}{|c|c|c|c|}
\hline Ligands with Bcl2 & Total Score & Polar & Global C Score \\
\hline Arachidic acid & 6.1543 & 3.0862 & 5 \\
\hline Butyric acid & 2.2670 & 3.0366 & 4 \\
\hline Capric acid & 4.8665 & 2.9358 & 5 \\
\hline Caproic acid & 2.9419 & 2.8628 & 4 \\
\hline Caprylic acid & 5.7833 & 2.9480 & 4 \\
\hline Cis Linoleic acid & 7.4224 & 1.4972 & 4 \\
\hline Cis Palmitoleic acid & 5.7277 & 2.7611 & 5 \\
\hline Conjugated Linolenic acid & 6.6013 & 1.4960 & 4 \\
\hline Hexadecanoic acid & 5.1495 & 2.7853 & 1 \\
\hline Myristic acid & 6.2331 & 2.9152 & 5 \\
\hline Pentadecanoic acid & 5.6325 & 2.7531 & 4 \\
\hline Stearic acid & 6.0341 & 2.7309 & 4 \\
\hline Trans oleic acid & 5.0402 & 2.9680 & 4 \\
\hline
\end{tabular}

Table 5. Scoring of the interaction between ligands and target protein CASPASE9.

\begin{tabular}{|c|c|c|c|}
\hline Ligands with Caspase9 & Total Score & Polar & Global C Score \\
\hline Arachidic acid & 6.8875 & 1.3989 & 5 \\
\hline Butyric acid & 2.3473 & 1.3681 & 3 \\
\hline Capric acid & 4.4618 & 1.4294 & 5 \\
\hline Caproic acid & 2.9855 & 1.3369 & 4 \\
\hline Caprylic acid & 4.0190 & 2.9371 & 5 \\
\hline Cis Linoleic acid & 6.2483 & 1.3660 & 2 \\
\hline Cis Palmitoleic acid & 6.1045 & 1.3977 & 4 \\
\hline Conjugated Linolenic acid & 6.7036 & 1.4162 & 5 \\
\hline Hexadecanoic acid & 6.9958 & 3.0576 & 4 \\
\hline Myristic acid & 5.3212 & 3.0721 & 4 \\
\hline Pentadecanoic acid & 6.5711 & 3.2523 & 3 \\
\hline Stearic acid & 6.7593 & 3.0924 & 5 \\
\hline Trans oleic acid & 6.6543 & 3.0257 & 3 \\
\hline
\end{tabular}


Table 6. Screening of ligands to check for Lipinski's Rule of Five.

\begin{tabular}{l|c|c|c|c|c} 
Ligands & Mass & $\begin{array}{c}\text { H Bond } \\
\text { Donor }\end{array}$ & $\begin{array}{c}\text { H Bond } \\
\text { Acceptor }\end{array}$ & Logp & Molar Refractivity \\
\hline Arachidic acid & 312.000000 & 1 & 2 & 5.550211 & 110.128281 \\
\hline Butyric acid & 88.000000 & 1 & 2 & 0.871100 & 22.543797 \\
\hline Capric acid & 172.000000 & 1 & 2 & 3.211699 & 50.245785 \\
\hline Caproic acid & 116.000000 & 1 & 2 & 1.651300 & 31.777792 \\
\hline Caprylic acid & 144.000000 & 1 & 2 & 2.431500 & 41.011787 \\
\hline Cis Linoleic acid & 266.000000 & 1 & 2 & 5.494400 & 82.376778 \\
\hline Cis Palmitoleic acid & 254.000000 & 1 & 2 & 5.328300 & 77.853775 \\
\hline Conjugated Linolenic acid & 280.000000 & 1 & 2 & 5.884500 & 86.993774 \\
\hline Hexadecanoic acid & 256.000000 & 1 & 2 & 5.552299 & 77.947777 \\
\hline Myristic acid & 228.000000 & 1 & 2 & 4.772099 & 68.713783 \\
\hline Pentadecanoic acid & 242.000000 & 1 & 2 & 5.162199 & 73.330780 \\
\hline Stearic acid & 284.000000 & 1 & 2 & 4.937631 & 99.194283 \\
\hline Trans oleic acid & 282.000000 & 1 & 2 & 6.108500 & 87.087776
\end{tabular}

\subsection{Need for complementary alternative medicine (CAM).}

Conventional cancer treatment therapies ve always imposed many challenges, including high financial expenses, multi-drug resistance, and increased toxicity levels to the human body. Hence, complementary alternative medicine (CAM) has become very important and has gained much attention due to less toxicity and increased specificity. Certain combinations of phytochemicals have shown a good synergistic effect in boosting the immune system, sensitizing cancer cells, inducing apoptosis and cell-cycle arrest, reducing cell proliferation, migration and invasion. One such study involved combining phytocompounds such as Resveratrol, Isoflavone, Curcumin, C-Phycocyanin, and Quercitin on breast cancer cells showed a good synergistic effect and can serve as potential CAM [21]. Anti-cancer efficacy of biologically synthesized AgNPs from endophytic fungus (isolated from Catharanthus roseus (Linn.), a medicinal plant) is also observed against lung cancer cell lines (A-549 cells) by inducing apoptosis and free radical scavenging under invitro conditions [22]. Research studies have also revealed that certain micro-organisms, when used in certain nanoparticles' biosynthesis, could alter apoptotic proteins' expression in cancer cells. One such study involves the synthesis of AgNPs (Silver nanoparticles) using endophytic bacteria isolated from the ornamental plant Pennisetum setaceum (drought-tolerant). These biologically synthesized AgNPs are found to alter the expression of Bax, Bcl2 (apoptotic proteins) and COX-2 (an inflammatory marker) and thus resulted in apoptosis of human breast cancer cells (MCF-7) under in-vitro conditions[23]. A similar effect in MCF-7 cells is also observed when AgNPs are biosynthesized from the aqueous extract using the seeds of Nigella sativa [24]. Dual-layered solid lipid nanoparticles (DL-SLN) made of pectin and skimmed milk powder loaded with soluble Curcumin is found to exhibit increased apoptotic activity in Human Colorectal Cancer cells (SW480 cells) [25].

Many studies have revealed that naturally available biomolecules could play a potentially important role against cancer cells. One such study is that the phytochemical extract of dried seed weed powder of Gelidiella acerosa, marine red algae, found to be rich in alkaloids, flavonoids, tannins, glycosides, fats, and oils in varied concentrations show antiproliferative activity against A549 (Lung cancer cell line) cells under in-vitro conditions [26]. Studies conducted to determine the concentration of biomolecules and its functional activity of buffalo milk when buffalo's diet included naturally available green feed such as alfa-alfa, showed higher concentration of short-chain acylcarnitines and $\delta$-Valerobetaine, which is shown to decrease the viability of human HCT116 and Cal 27 cancer cells [27]. 
Studies based on Skim milk powder (SMP) have also proven useful against cancer. Wand A. et al. have shown that Caffeic acid phenethly ester (CAPE) loaded in SMP microcapsules exhibits antiproliferative activity against human cancerous colon cells compared to free CAPE as it has very poor water solubility and bioavailability [28]. Research studies conducted by Yuan G. et al., have shown that milk intake reduced the risk of luminal breast cancer, whereas, in non-luminal breast cancer, milk is shown to be a very effective protective factor [29].

Obesity has always been a major concern for serious health hazards in humans and is also one of the important risk factors for cancer. Studies have shown that metastatic cancer cells' survival is highly supported by fat tissue, which allows tumors to form at distant sites. Lifestyle interventions and the evolution of new therapies can help prevent the communication between tumor and fat cells [30]. Highly efficient inhibitory effects on cancer cells have also been demonstrated by some dietary components such as isothiocyanates, isoflavones, lycopene, catechins, cucurbitacin B, and capsaicin [31]. The intestinal microbiome produces fermentation products such as short-chain fatty acids (SCFA) that serve as a rich source of energy supply to the gut's epithelial cells. Reduction in the gut bacteria producing SCFA can result in inflammation, which can initiate tumor progression through numerous pathways such as activation of nuclear factor-kappa $\mathrm{B}(\mathrm{NF}-\mathrm{\kappa B})$ pathway, mutations in tumor suppressor gene Tp53, and by enhancing DNA damage. Hence, an optimal amount of SCFA is necessary to protect diabetic people from tumorigenesis [32].

Linoleic acid, combined with Lactoferrin, is found to inhibit JAK2/STAT3-related pathway, which inhibits tumor growth in mice [33]. The incidence of ER-/PR- breast cancer is found to decrease significantly when fermented dairy products such as ghee are consumed on a long-term basis [34]. From these studies, we understand the importance of naturally available biomolecules and their role in targeted cancer therapies, and hence the fatty acids present in ghee can serve the purpose.

\section{Conclusions}

The interaction between the fatty acids present in the home-made ghee and the target proteins (AIF, PTEN, BAX, BCL2, and CASPASE9) highlights the importance and efficiency of the home-made ghee in providing anti-cancer effects. It also suggests the need for performing more in vitro studies for further confirmations. This study also provides evidence that consuming home-made ghee both as a part of the diet and also on an empty stomach might increase the preventive possibilities for the incidence of cancer, and also ghee consumption might reduce a person's susceptibility for inheriting some of the cancer types like breast and colon cancer which needs confirmation through further in vitro research.

\section{Funding}

Ministry of Science and Technology, Department of Science and Technology (KIRAN Division) (GoI), New Delhi. (Ref No. DST/WOSB/ 2018/1583-HFN (G).

\section{Acknowledgments}

The authors gratefully acknowledge the Ministry of Science and Technology, Department of Science and Technology (KIRAN Division) (GoI), New Delhi. (Ref No. DST/WOSB/ 
2018/1583-HFN (G)). The authors are also thankful to B.S. Abdur Rahman Institute of Science \& Technology, Chennai, for providing research facilities in the School of Life Sciences.

\title{
Conflicts of Interest
}

\author{
The authors declare no conflict of interest.
}

\section{References}

1. Chinnadurai, K.; Kanwal, H.K.; Tyagi, A.K.; Stanton, C.; Ross, P. High conjugated linoleic acid enriched ghee (clarified butter) increases the antioxidant and antiatherogenic potency in female Wistar rats. Lipids in health and disease 2013, 12,https://doi.org/10.1186/1476-511X-12-121.

2. Ahmad, N.; Saleem, M. Raman spectroscopy based characterization of desi ghee obtained from buffalo and cow milk. International Dairy Journal 2019, 89, 119-128,https://doi.org/10.1016/j.idairyj.2018.08.013.

3. Cichosz, G.; Czeczot, H.; Bielecka, M. The anticarcinogenic potential of milk fat. Ann Agric Environ Med. 2020, 27, 512-518, http://dx.doi.org/10.26444/aaem/116095.

4. Dorni, C.; Sharma, P.; Saikia, G.; Longvah, T. Fatty acid profile of edible oils and fats consumed in India. Food Chemistry 2018, 238, 9-15,http://dx.doi.org/10.1016/j.foodchem.2017.05.072.

5. Bali, S.; Utaal, M.S. Ghee, the much-aligned cooking medium, now slowly, reclaiming its therapeutic reputation. Int J Sci Rep 2019, 5, 370-372,http://dx.doi.org/10.18203/issn.2454-2156.IntJSciRep20195303.

6. Joshi, D.; Adhikari, N. Benefit of Cow Urine, Milk, Ghee, Curd, and Dung Versus Cow Meat: Mini Review. 2019, 3, 169-175,http://dx.doi.org/10.31080/ASPS.2019.03.0360.

7. Hanahan, D.; Weinberg, R.A. Hallmarks of cancer: the next generation. Cell 2011, 144, 646-674, http://dx.doi.org/10.1016/j.cell.2011.02.013.

8. Devlin, E.J.; Denson, L.A.; Whitford, H.S. Cancer Treatment Side Effects: A Meta-analysis of the Relationship Between Response Expectancies and Experience. Journal of pain and symptom management 2017, 54, 245-258,https://doi.org/10.1016/j.jpainsymman.2017.03.017.

9. Jamal Moideen, M.M.; Alqahtani, A.; Venkatesan, K.; Ahmad, F.; Krisharaju, K.; Gayasuddin, M.; Shaik, R.A.; Ibraheem, K.M.M.; Salama, M.E.L.d.M.; Abed, S.Y. Application of the Box-Behnken design for the production of soluble curcumin: Skimmed milk powder inclusion complex for improving the treatment of colorectal cancer. Food Science \& Nutrition 2020, 8, 6643-6659,https://doi.org/10.1002/fsn3.1957.

10. van Slambrouck, S.; Daniels, A.L.; Hooten, C.J.; Brock, S.L.; Jenkins, A.R.; Ogasawara, M.A.; Baker, J.M.; Adkins, G.; Elias, E.M.; Agustin, V.J.; Constantine, S.R.; Pullin, M.J.; Shors, S.T.; Kornienko, A.; Steelant, W.F.A. Effects of crude aqueous medicinal plant extracts on growth and invasion of breast cancer cells. Oncol Rep 2007, 17, 1487-1492,https://doi.org/10.3892/or.17.6.1487.

11. Newman, D.J.; Cragg, G.M. Natural Products as Sources of New Drugs over the Last 25 Years. Journal of Natural Products 2007, 70, 461-477.

12. Susmita, M.; Santu, B.; Mrinal, K.G.; Sibabrata, M.; Siddhartha, R.; Chitra, M. Natural Products: Promising Resources for Cancer Drug Discovery. Anti-cancer Agents in Medicinal Chemistry 2012, 12, 49-75, https://doi.org/10.2174/187152012798764697.

13. Priya, S.; Kumar, N.S.; Hemalatha, S. Antiviral phytocompounds target envelop protein to control Zika virus. Computational Biology and Chemistry 412,https://doi.org/10.1016/j.compbiolchem.2018.08.008.

14. Lutfiya, A.S.; Priya, S.; Manzoor, M.A.P.; Hemalatha, S. Molecular docking and interactions between vascular endothelial growth factor (VEGF) receptors and phytochemicals: An in-silico study. Biocatalysis and Agricultural Biotechnology 2019, 22, https://doi.org/10.1016/j.bcab.2019.101424.

15. Arpudhamary, V.; Priya, S.; Manzoor, M.A.P.; Mubarakali, D.; Hemalatha, S. Apoptotic-inducing factor 1 (AIF1) plays a critical role in cembranoid mediated apoptosis to control cancer: Molecular docking and dynamics study. Biocatalysis and Agricultural Biotechnology 2019, 22, https://doi.org/10.1016/j.bcab.2019.101343.

16. Pal, U.K.; Mandal, P.; Rao, V.K.; Das, C.D. Quality and Utility of Goat Milk with Special Reference to India: An Overview. Asian Journal of Animal Sciences 2011, 5, 5663,https://doi.org/10.3923/ajas.2011.56.63.

17. Haenlein, G.F.W. Goat milk in human nutrition. Small Ruminant Research 2004, 51, 155-163, https://doi.org/10.1016/j.smallrumres.2003.08.010.

18. Bhattarai R.R. Importance of goat milk.J. Food Sci. Technol2012, 7, 107111,https://doi.org/10.3126/jfstn.v7i0.11209.

19. Narayanan, A.; Baskaran, S.A.; Amalaradjou, M.A.; Venkitanarayanan, K. Anticarcinogenic Properties of Medium Chain Fatty Acids on Human Colorectal, Skin and Breast Cancer Cells in Vitro. International Journal of Molecular Sciences 2015, 16, 5014-27,https://doi.org/10.3390/ijms16035014.

20. Arab, A.; Akbarian, S.A.; Ghiyasvand, R.; Miraghajani, M. The effects of conjugated linoleic acids on breast cancer: A systematic review. Adv Biomed Res2016, 5, 115-115, https://doi.org/10.4103/2277-9175.185573. 
21. Rizeq, B.; Gupta, I.; Ilesanmi, J.; AlSafran, M.; Rahman, M.D.M.; Ouhtit, A. The Power of Phytochemicals Combination in Cancer Chemoprevention. Journal of Cancer 2020, 11, 4521-4533, https://doi.org/10.7150/jca.34374.

22. Akther, T.; Vabeiryureilai, M.; Nachimuthu Senthil, K.; Davoodbasha, M.; Srinivasan, H. Fungal-mediated synthesis of pharmaceutically active silver nanoparticles and anti-cancer property against A549 cells through apoptosis. Environmental Science and Pollution Research 2019, 26, 13649-13657, https://doi.org/10.1007/s11356-019-04718-w.

23. Shariq Ahmed, M.; Soundhararajan, R.; Akther, T.; Kashif, M.; Khan, J.; Waseem, M.; Srinivasan, H. Biogenic AgNPs synthesized via endophytic bacteria and its biological applications. Environmental Science and Pollution Research 2019, 26, 26939-26946, https://doi.org/10.1007/s11356-019-05869-6.

24. Rohini, B.; Akther, T.; Waseem, M.; Khan, J.; Kashif, M.; Hemalatha, S. AgNPs from Nigella sativa Control Breast Cancer: An In Vitro Study. Journal of environmental pathology, toxicology and oncology : official organ of the International Society for Environmental Toxicology and Cancer 2019, 38, 185-194, https://doi.org/10.1615/JEnvironPatholToxicolOncol.2019027318.

25. Mohamed, J.M.; Alqahtani, A.; Ahmad, F.; Krishnaraju, V.; Kalpana, K. Pectin co-functionalized dual layered solid lipid nanoparticle made by soluble curcumin for the targeted potential treatment of colorectal cancer. Carbohydrate Polymers 2021, 252, https://doi.org/10.1016/j.carbpol.2020.117180.

26. Fazeela Mahaboob Begum, S.M.; Hemalatha, S. Phytoconstituents from Gelidiella acerosa induce apoptosis by regulating Bax, Bcl2 expression in A549 cells. Biocatalysis and Agricultural Biotechnology 2020, 29, https://doi.org/10.1016/j.bcab.2020.101757.

27. Salzano, A.; Neglia, G.; D’Onofrio, N.; Balestrieri, M.L.; Limone, A.; Cotticelli, A.; Marrone, R.; Anastasio, A.; D’Occhio, M.J.; Campanile, G. Green feed increases antioxidant and antineoplastic activity of buffalo milk: A globally significant livestock. Food Chemistry 2021, 344, https://doi.org/10.1016/j.foodchem.2020.128669.

28. Wang, A.; Leible, M.; Lin, J.; Weiss, J.; Zhong, Q. Caffeic Acid Phenethyl Ester Loaded in Skim Milk Microcapsules: Physicochemical Properties and Enhanced In Vitro Bioaccessibility and Bioactivity against Colon Cancer Cells. J Agric Food Chem 2020, 68, 14978-14987,https://doi.org/10.1021/acs.jafc.0c05143.

29. Yuan, G.; Zhang, J.; Ren, Y.; Ding, W.; Du, Y.; Zhang, L.; Shao, J. Dietary effects on breast cancer molecular subtypes, a 1:2 paired case-control study. Food Science \& Nutrition 2020, 8, 5545-5549, https://doi.org/10.1002/fsn3.1866.

30. Annett, S.; Moore, G.; Robson, T. Obesity and Cancer Metastasis: Molecular and Translational Perspectives. Cancers 2020, 12,https://doi.org/10.3390/cancers12123798.

31. Ranjan, A.; Ramachandran, S.; Gupta, N.; Kaushik, I.; Wright, S.; Srivastava, S.; Das, H.; Srivastava, S.; Prasad, S.; Srivastava, S.K. Role of Phytochemicals in Cancer Prevention. International Journal of Molecular Sciences 2019, 20,https://doi.org/10.3390/ijms20204981.

32. Yang, Q.; Ouyang, J.; Sun, F.; Yang, J. Short-Chain Fatty Acids: A Soldier Fighting Against Inflammation and Protecting From Tumorigenesis in People With Diabetes. Frontiers in Immunology 2020, 11, https://doi.org/10.3389/fimmu.2020.590685.

33. Li, H.; Yao, Q.; Min, L.; Huang, S.; Wu, H.; Yang, H.; Fan, L.; Wang, J.; Zheng, N. The Combination of Two Bioactive Constituents, Lactoferrin and Linolenic Acid, Inhibits Mouse Xenograft Esophageal Tumor Growth by Downregulating Lithocholyltaurine and Inhibiting the JAK2/STAT3-Related Pathway. ACS Omega 2020, 5, 20755-20764, https://doi.org/10.1021/acsomega.0c01132.

34. Kaluza, J.; Komatsu, S.; Lauriola, M.; Harris, H.R.; Bergkvist, L.; Michaëlsson, K.; Wolk, A. Long-term consumption of non-fermented and fermented dairy products and risk of breast cancer by estrogen receptor status - $\quad$ Population-based prospective cohort study. Clin Nutr2020, in press,https://doi.org/10.1016/j.clnu.2020.09.013. 